\section{THU0031 FILGOTINIB TREATMENT PROVIDES RAPID AND SUSTAINED REDUCTION OF INFLAMMATORY BIOMARKERS IN MODERATE TO SEVERE PSORIATIC ARTHRITIS (PSA) PATIENTS}

Dafna D Gladman ${ }^{1}$, Rene Galien ${ }^{2}$, Corrine Jamoul ${ }^{3}$, Mona Trivedi ${ }^{4}$ Neelufar Mozaffarian ${ }^{4}$, Ohkyu Yoon ${ }^{4}$, Wendy Jiang ${ }^{4}$, Jiyun Kim ${ }^{4} .{ }^{1}$ University of Toronto and Krembil Research Institute, Toronto Western Hospital, Toronto, Canada; ${ }^{2}$ Galapagos SASU, Romainville, France; ${ }^{3}$ Galapagos NV, Mechelen, Belgium; ${ }^{4}$ Gilead Sciences Inc, Foster City, United States of America

Background: PsA is a chronic inflammatory musculoskeletal disease characterized by joint and skin inflammation, enthesitis, dactylitis, and nail lesions. In the recent EQUATOR study, filgotinib (FIL), an oral, selective Janus kinase 1 (JAK1) inhibitor, produced significant and sustained improvements on the signs and symptoms of PSA compared to placebo (PBO). ${ }^{1}$ As targeted JAK1 inhibition by FIL has potential to simultaneously block multiple inflammatory pathways, we analyzed biomarker samples from EQUATOR using exploratory multiplex biomarker panels.

Objectives: To evaluate the impact of targeted JAK1 inhibition on the circulating biomarkers in patients with active PsA.

Methods: Design and results of the EQUATOR study - a 16-week, double-blind, multicenter, phase 2 study in subjects with active PsA (NCT03101670) - have been published. ${ }^{1}$ Subjects were randomized 1:1 to $200 \mathrm{mg}$ FIL $(n=65)$ or PBO $(n=66)$ once daily. Serum samples (FIL $n=60$ and PBO $\mathrm{n}=61$ ) were collected at baseline (BL), week 1 , week 4 , and week 16, and analyzed using 135 multiplex biomarker screening panels. Biomarker changes from BL were analyzed on all time-paired subject data and reported for weeks 1,4 , and 16 .

Results: FIL treatment produced significant reductions serum concentration of multiple biomarkers associated with PsA disease activity. We identified 4 clusters of biomarker response based on the kinetics and magnitude of changes from BL (Figure); Cluster 1: rapid, substantial and sustained reduction ( $>25 \%$ from $\mathrm{BL}$ by week 1 ); Cluster 2 : rapid and moderate reduction $(5 \%-15 \%$ from $\mathrm{BL}$ by week 1); Cluster 3: delayed reduction (significant reduction by week 4); and Cluster 4: moderate increase $(5 \%-$ $10 \%$ from BL). Although initial grouping was based on the speed and magnitude of biomarker response, a number of biomarkers formed subclusters representing discrete biological functions. Cluster 1 included 2 acute phase reactants (CRP, SAA) while Cluster 2 included inflammatory mediators (TNFR1, CXCL10, IL-12/-23; CXCL10 and IL-12/-23 reached full reduction by week 1). Cluster 3 was the largest group and represented biomarkers of matrix remodeling (MMP1, TIMP1), cell adhesion (ICAM-1, E-selectin), and angiogenesis (VEGF-A). Cluster 4 had biomarkers related to diverse functions (Eotaxin, CEA, adiponectin).

Conclusion: In patients with active PsA, FIL treatment significantly decreased levels of circulating biomarkers, including acute-phase reactants, proinflammatory cytokines and chemokines, and various adhesion molecules. These data support the idea that FIL treatment results in rapid and sustained reduction of the inflammatory milieu present in autoimmune inflammatory diseases, consistent with efficacy previously reported for this study. ${ }^{1}$

Figure. Heatmap with $\%$ change and $p$ values.

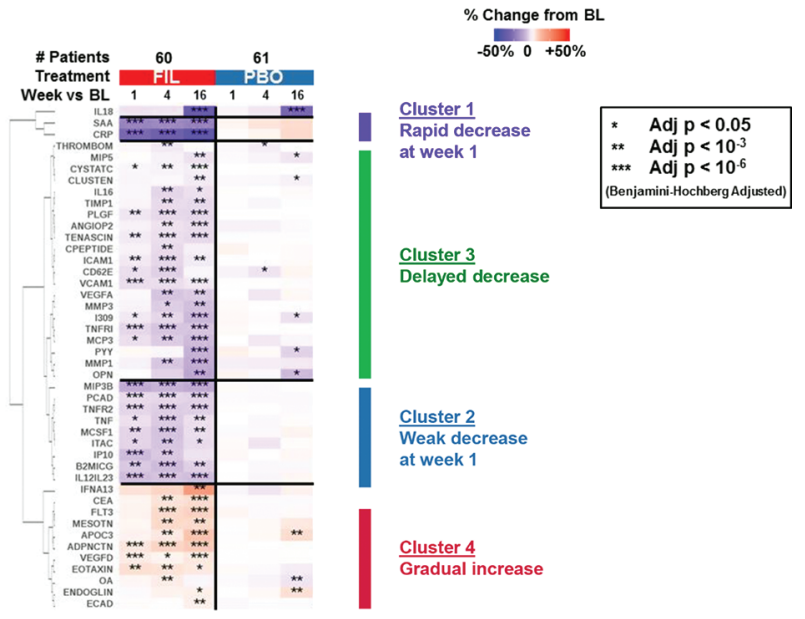

REFERENCE:

[1] Mease PJ, et al. Lancet. 2018;392:2367-2377.

Disclosure of Interests: Dafna D Gladman Grant/research support from: AbbVie, Amgen, Celgene, Lilly, Novartis, Pfizer, and UCB, Consultant for: AbbVie, Amgen, BMS, Celgene, Galapagos, Gilead, Janssen, Lilly, Novartis, Pfizer, and UCB, Rene Galien Shareholder of: Gilead Sciences, Inc., Employee of: Gilead Sciences, Inc., Corrine Jamoul Employee of: Galapagos NV, Mona Trivedi Shareholder of: Amgen and Gilead Sciences, Inc. Employee of: Gilead Sciences, Inc., Neelufar Mozaffarian Shareholder of: Gilead, Employee of: Gilead, OhKyu Yoon Shareholder of: Gilead Sciences, Inc., Amgen, Employee of: Gilead Sciences, Inc., Amgen, Wendy Jiang Shareholder of: Gilead Sciences, Inc., Employee of: Gilead Sciences, Inc., JiYun Kim Shareholder of: Gilead Sciences, Inc., Employee of: Bayer Healthcare, LLC, Gilead Sciences, Inc.

DOI: 10.1136/annrheumdis-2019-eular.2464

\section{THU0032 DYNAMICS OF ADIPOCYTOKINES IN PATIENTS WITH EARLY RHEUMATOID ARTHRITIS IN DIFFERENT TREATMENT REGIMENS}

Yulia Gorbunova, Tatiana Popkova, Evgeny Nasonov. Nasonova Research Institute of Rheumatology, Moscow, Russian Federation

Background: Adipose tissue is a highly active endocrine organ that produces a large number of various pro-inflammatory cytokines and adipocytokines (adiponectin, leptin, etc.) involved in the pathogenesis of rheumatoid arthritis (RA). The relationship of adipokines with activity markers and inflammation in RA and the effect of antirheumatic therapy on adipocytokine levels in patients with early RA is discussed.

Objectives: To assess the dynamics of adipocytokine levels (adiponectin leptin) in patients with early RA (initial and after 24 weeks) using various treatment regimens: methotrexate monotherapy (MT) and combination therapy (MT + adalimumab (ADA).

Methods: The study included 47 patients with early RA (criteria ACR/ EULAR, 2010.), 57 [46.5; 62.0] years, disease duration 6.0 [5.5;15.5] months, seropositive for IgM RF and anti-CCP, with highly active RA (DAS28 5,5 [5,1; 5,9]; SDAl 32,4 [22,4; 41,7], CDAl 29,0 [19,7; 39,5]). At study entry, all patients received methotrexate (MTX) (10 [10-15] mg/ week), after 12 weeks, with inefficiency of MT, adalimumab (ADA) was administered $40 \mathrm{mg} 1$ time in 2 weeks. Serum concentration of adipokines (adiponectin, leptin) was measured with ELISA.

Results: By the 24th week of therapy, $23(49 \%)$ patients with early RA received a combination therapy (adalimumab and methotrexate), $24(51 \%)$ patients with RA received methotrexate monotherapy. In patients with RA, after 24 weeks, a significant decrease in adiponectin levels by 44 and $49 \%$ and an increase in the concentration of leptin by 39 and $43 \%$ were observed both against the background of MT monotherapy and with the use of combination therapy ( $p<0.05$ in all cases). By the end of the study, adiponectin levels were higher in the MT monotherapy group, and leptin in the combination therapy group.

Table 1:

\begin{tabular}{lcccccc}
\hline & \multicolumn{3}{c}{ Monotherapy MT $(\mathrm{n}=24)$} & \multicolumn{3}{c}{$\begin{array}{c}\text { Combination therapy MT+ADA } \\
(\mathrm{n}=23)\end{array}$} \\
\cline { 2 - 7 } & 0 point & 24 weeks & $\Delta, \%$ & 0 point & 24 weeks & $\Delta, \%$ \\
\hline Adiponectin, & 23,0 & $12,8[8,0 ; 19,6]^{\star} /$ & -44 & $20,0[13 ; 34]$ & $10,2[7,0 ; 12,1]$ & -49 \\
ng/ml & {$[12,6 ; 77,0]$} & $* \star$ & & & $*$ & \\
Leptin, ng/ml & 15,6 & $21,7[12,5 ; 40,4]$ & +39 & 20,0 & 28,1 & +43 \\
& {$[7,8 ; 27,5]$} & $* / * \star$ & & {$[11 ; 39,2]$} & {$[12,4 ; 58,3]^{\star}$} & \\
\hline
\end{tabular}

* $-p<0,05$ reliability of differences in factors before treatment and after 6 months (Wilcoxon); ${ }^{* *}-p<0.05$ difference in the indices between the groups by the 6 th month of therapy; $\Delta, \%$-the difference between the indices between the groups by the 6th month of therapy.

Conclusion: After 24 weeks of therapy, the adipocytokine levels in the two groups changed: the adiponectin concentration decreased, and the leptin level increased. MT monotherapy and combination therapy (MT + ADA) acted on changes in adipocytokine levels unidirectionally, although the changes were more pronounced in patients on combination therapy. Disclosure of Interests:

None declared

DOI: 10.1136/annrheumdis-2019-eular.7621 